\title{
Spontaneous Delayed Migration/Shortening of the Pipeline Embolization Device: Report of 5 Cases
}

\author{
N. Chalouhi, S.I. Tjoumakaris, L.F. Gonzalez, D. Hasan, P.J. Pema, G. Gould, R.H. Rosenwasser, and P.M. Jabbour
}

\begin{abstract}
SUMMARY: Five patients were found to have spontaneous delayed migration/shortening of their Pipeline Embolization Devices on follow-up angiography. The device migrated proximally in 4 patients and distally in 1 patient. One patient had a subarachnoid hemorrhage and died as a result of migration of the Pipeline Embolization Device, and another patient presented with complete MCA occlusion and was left severely disabled. Mismatch in arterial diameter between inflow and outflow vessels was a constant finding. Migration of the Pipeline Embolization Device was managed conservatively, with additional placement of the device, or with parent vessel occlusion. Obtaining complete expansion of the embolization device by using a longer device, increasing vessel coverage, using adjunctive aneurysm coiling, and avoiding dragging and stretching of the device are important preventive measures. Neurointerventionalists should be aware of this potentially fatal complication and take all necessary preventive measures.
\end{abstract}

ABBREVIATIONS: PED = Pipeline Embolization Device; SHA = superior hypophyseal artery

$\mathrm{T}$ he Pipeline Embolization Device (PED; Covidien/ev3, Irvine, California) is a dedicated flow diverter designed to treat intracranial aneurysms. Recent series have shown the PED to be a reasonably safe and effective treatment. ${ }^{1-5}$ The risks for intracranial hemorrhage, delayed aneurysm rupture, and thromboembolic events have been major concerns with this approach. ${ }^{1-3,6,7}$ Additional complications are becoming evident, as the PED is increasingly used for an expanding population of patients with cerebral aneurysms. In this study, we report 5 cases of patients with spontaneous delayed migration or shortening of the PED and discuss the possible cause, implications, and management of this serious and potentially fatal complication.

\section{MATERIALS AND METHODS}

Five patients treated with the PED in whom spontaneous delayed migration or shortening of their devices developed were prospectively identified. During the same period (from November 2010January 2013), a total of 155 patients were treated with the PED at

Received January 31, 2013; accepted after revision March 20.

From the Department of Neurosurgery (N.C., S.I.T., L.F.G., G.G., R.H.R., P.M.J.), Thomas Jefferson University and Jefferson Hospital for Neuroscience, Philadelphia, Pennsylvania; Department of Neurosurgery (D.H.), University of lowa, lowa City, lowa; and Department of Radiology (P.J.P.), Riverside Methodist Hospital, Columbus, Ohio.

Please address correspondence to Pascal M. Jabbour, MD, Associate Professor, Department of Neurological Surgery, Division of Neurovascular Surgery and Endovascular Neurosurgery, Thomas Jefferson University Hospital, 901 Walnut St, 3rd Floor, Philadelphia, PA 19107; e-mail: pascal.jabbour@jefferson.edu

http://dx.doi.org/10.3174/ajnr.A3632 our institution. The 5 cases of PED migration occurred late during the study period (second half of the cohort).

PEDs were deployed through a Marksman microcatheter (ev3, Irvine, California) by use of a triaxial guide-catheter system. The maximal diameter in the predicted landing zone was determined, and the PED was sized according to the width of the inflow vessel to avoid any endoleak. The expansion of the PED was documented under fluoroscopy or with additional DynaCT angiography (Siemens, Erlangen, Germany) at the operator's discretion.

\section{Representative Case Patients}

Patient 1. A 50-year-old man with schizophrenia presented with an unruptured left MCA (M1) aneurysm discovered during work-up for severe cervical myelopathy. The aneurysm measured $12.7 \times 11.6 \mathrm{~mm}$; the diameter of the inflow vessel was $3.2 \mathrm{~mm}$, and the diameter of the outflow vessel was $2.5 \mathrm{~mm}$. Given the aneurysm size, wide-neck morphologic feature, and the patient's categoric refusal of open surgery, the decision was made to treat the aneurysm with the PED. The patient was pretreated with aspirin and clopidogrel for 10 days. The patient was given a bolus of heparin, and activated clotting time was maintained at 2-3 times the baseline throughout the procedure. With use of a triaxial system, a $3 \times 16-\mathrm{mm}$ PED was deployed across the neck of the aneurysm, extending from the proximal superior division of the M2 segment to the mid-M1 segment (Fig 1). A control cerebral angiogram was performed and showed stasis within the aneurysm. Five months later, the patient presented to the emergency 

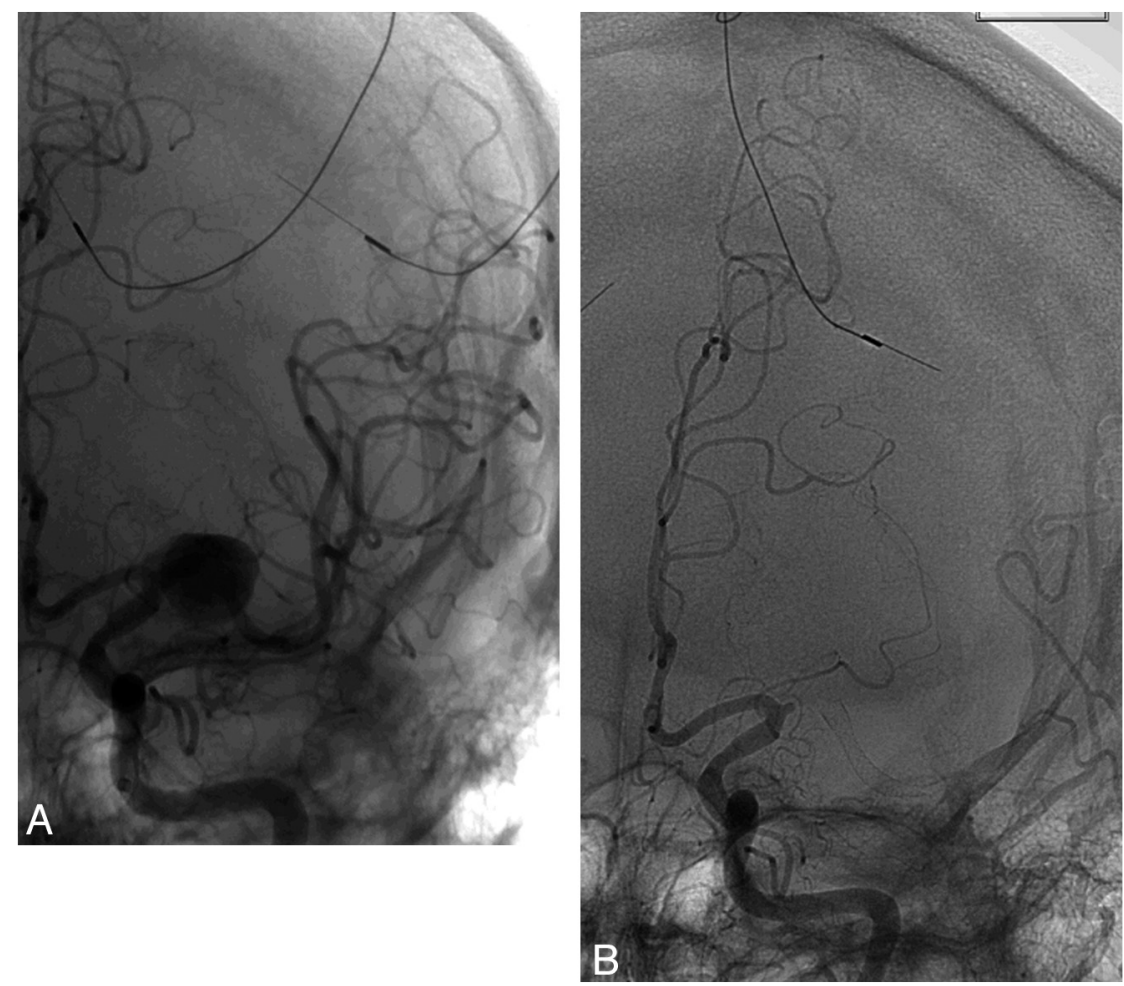

FIG 1. A, Angiogram after deployment of a PED extending from the proximal superior division of the $M 2$ segment to the mid-M1 segment. $B$, Angiogram showing complete MCA occlusion 5 months later with distal PED migration.

department with an acute onset of right-sided hemiplegia. He had not been compliant with the antiplatelet therapy. A noncontrast head CT scan result was negative for hemorrhage, and CT angiography demonstrated complete occlusion of the M1. CT perfusion revealed a large area at risk, and the patient was transferred to the angiography suite for mechanical thrombectomy. A left ICA injection showed that the M1 segment of the MCA was completely occluded. It is interesting to note that the PED had migrated distally, with the proximal end of the device now within the aneurysm sac. There was no evidence of filling of the aneurysm. The procedure was aborted at this point because it was felt that blind navigation through the aneurysm sac would carry a high risk for aneurysm perforation. The patient remained hemiplegic and was discharged to rehabilitation.

Patient 2. A 46-year-old woman presented to our institution with a 1-year history of visual loss. A visual field test revealed right-sided homonymous hemianopsia, and MRA showed a giant left supraclinoid ICA aneurysm. Flow diversion was thought to be the most durable treatment of the aneurysm. The patient was premedicated with aspirin and clopidogrel (Plavix). On DSA, the aneurysm measured $2.8 \times 2.3 \mathrm{~cm}$ with no distinct neck; the inflow zone was slightly distal to the ophthalmic artery, and the outflow zone was posterolateral within the aneurysm. There was delayed filling of the anterior and middle cerebral arteries caused by aneurysmal blood steal. A $4.25 \times$ 30-mm PED was deployed from the mid-M1 segment (diameter, $1.9 \mathrm{~mm}$ ) to the proximal cavernous ICA (diameter, 3.8 $\mathrm{mm}$ ) (Fig 2). Control angiography showed significant contrast stasis in the entire aneurysm with excellent filling of the ante- rior and middle cerebral arteries. The patient was neurologically intact after the procedure, but on postoperative day 3 she experienced right-sided hemiplegia with severe headaches. A noncontrast head CT scan was performed emergently and showed extensive subarachnoid hemorrhage. She underwent emergent reversal of antiplatelet therapy with placement of a right frontal ventriculostomy and was taken for subsequent angiography. On DSA, there was evidence of proximal PED migration, with displacement of the distal limb of the device now located centrally within the aneurysm sac and diverting the flow jet directly into the dome of the aneurysm. There was also evidence of a new rupture site on the aneurysmal dome (facing the distal end of the device). Several coils were deployed inside the aneurysm, and the decision was made to deconstruct the parent artery to prevent a new catastrophic hemorrhage. The left ICA was successfully occluded with a combination of coils and Onyx 34 (ev3). A control cerebral angiogram showed no filling of the left ICA and aneurysm. Despite maximal medical intervention, the patient experienced uncontrollable intracranial pressure and rapidly progressed to brain death.

Patient 3. A 41-year-old woman presented to our institution for evaluation and treatment of 2 incidentally discovered aneurysms arising from the left superior hypophyseal segment and the left cavernous segment of the ICA. The aneurysm of the superior hypophyseal artery (SHA) measured $6.43 \times 6.97 \mathrm{~mm}$ with a neck of $3.6 \mathrm{~mm}$, and the cavernous aneurysm measured $2.97 \times 3.63 \mathrm{~mm}$ with a $3-\mathrm{mm}$ neck. The diameter of the petrous segment of the ICA was $4.1 \mathrm{~mm}$, and the diameter of the paraclinoid segment was $2.7 \mathrm{~mm}$. The decision was made to treat both aneurysms with a flow-diversion technique by use of the PED. The patient was prescribed aspirin and clopidogrel. With use of a triaxial system, a $4 \times 25$-mm PED was deployed across the aneurysms without difficulty, extending from the paraclinoid segment of the ICA to the petrous segment (Fig 3). Control angiography demonstrated adequate PED placement with contrast stasis in the SHA aneurysm. DynaCT was subsequently performed, demonstrating adequate stent apposition to the vessel wall with covering of the necks of both aneurysms. The procedure and postoperative course were uneventful, and the patient was discharged on the following day. Follow-up angiography 4 months later showed that the distal portion of the PED had migrated slightly proximally, with the neck of the SHA aneurysm no longer covered by the device. There was complete filling of the SHA aneurysm, with no contrast stasis. The cavernous aneurysm had completely resolved. The patient was scheduled for subsequent embolization.

Patient 4. A 61-year-old woman with increasing retro-orbital pain from a large right cavernous ICA aneurysm underwent un- 

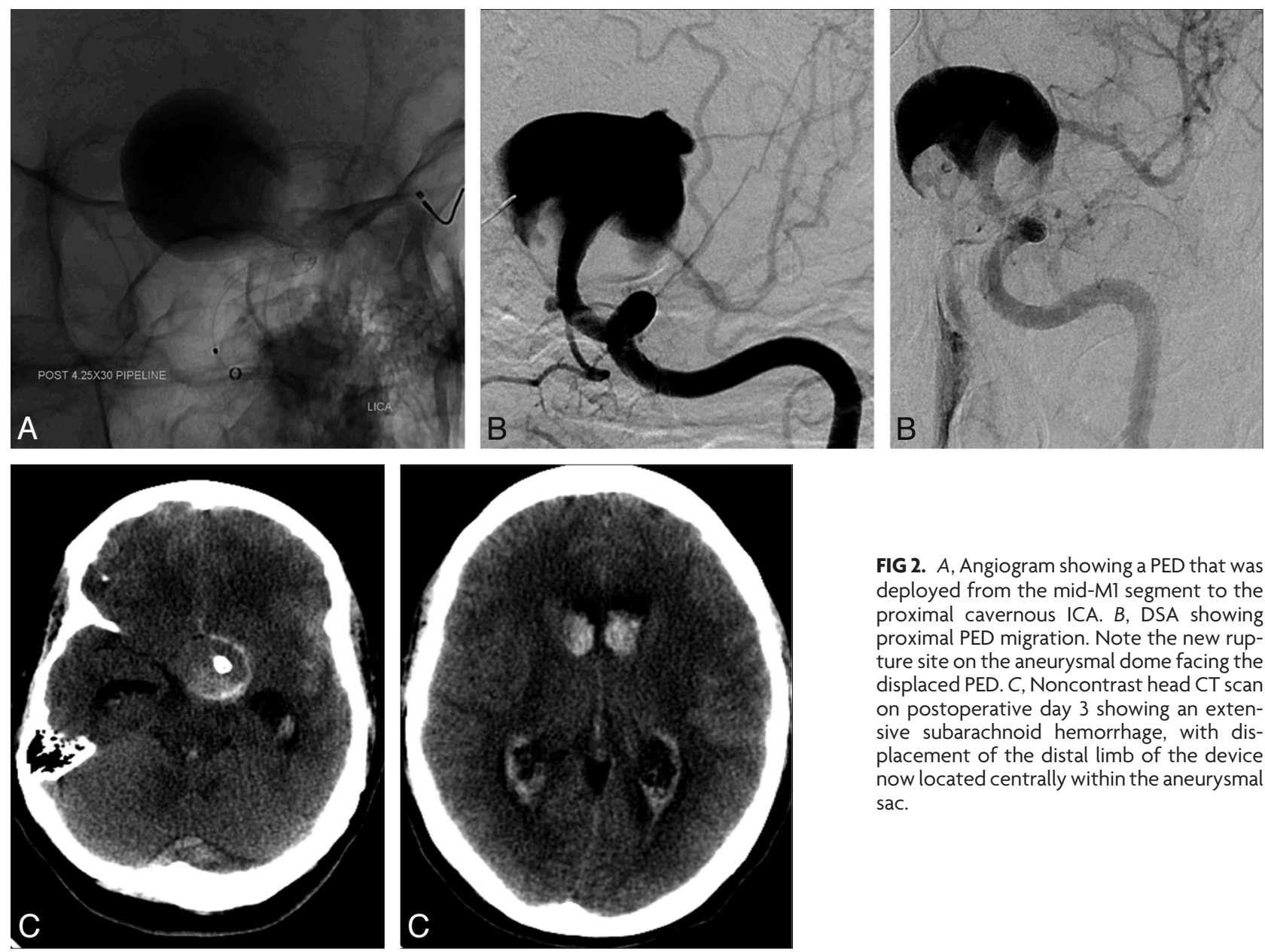

FIG 2. A, Angiogram showing a PED that was deployed from the mid-Ml segment to the proximal cavernous ICA. B, DSA showing proximal PED migration. Note the new rupture site on the aneurysmal dome facing the displaced PED. C, Noncontrast head CT scan on postoperative day 3 showing an extensive subarachnoid hemorrhage, with displacement of the distal limb of the device now located centrally within the aneurysmal sac.
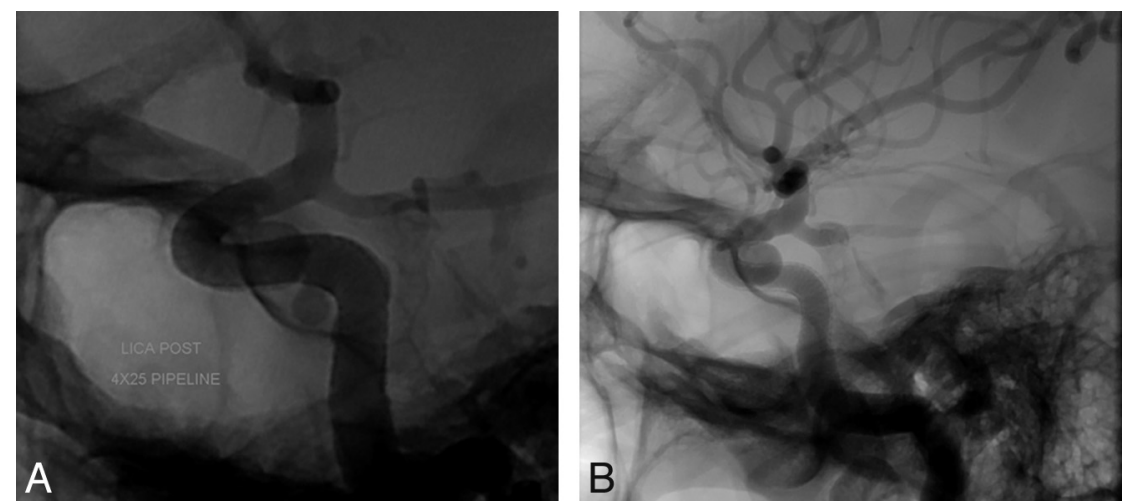

FIG 3. A, A PED is deployed across 2 aneurysms extending from the petrous segment to the paraclinoid segment of the ICA. B, Follow-up angiography 4 months later showing that the distal portion of the PED had migrated slightly proximally, with the neck of the SHA aneurysm no longer covered by the device.

eventful treatment of her aneurysm with the PED (Fig 4). There was significant mismatch in arterial diameter $(>1 \mathrm{~mm})$ between the inflow and outflow vessels. At 5 months after the procedure, the patient's symptoms recurred. On the 6-month follow-up angiography, there was proximal PED migration, with the distal end of the device projecting directly into the aneurysm and creating a jet of contrast against the aneurysm sac. A second, overlapping PED was successfully deployed within the first PED to cover the neck of the aneurysm. Six weeks later, the patient's symptoms had completely resolved and a follow-up CT angiogram showed complete obliteration of the aneurysm.

Patient 5. A 71-year-old woman presented for treatment of a symptomatic aneurysm of the left cavernous ICA measuring $16.5 \mathrm{~mm}$. The diameter of the inflow vessel was $4.4 \mathrm{~mm}$, and the diameter of the outflow vessel was $4.2 \mathrm{~mm}$. It was decided to treat the aneurysm with flow diversion. A $4.5 \times 18$-mm PED was deployed across the aneurysm without difficulty, extending from the immediate pregenu portion to the midvertical segment of the cavernous ICA. A control angiogram demonstrated contrast stasis within the aneurysm, with adequate stent apposition to the vessel wall. A subsequent angiogram 1 day later showed complete occlusion of the aneurysm. The postoperative course was uneventful, and the patient was discharged neurologically intact. She remained asymptomatic and returned 7 months later for follow-up angiography. On DSA, there was complete filling of the aneurysm sac and the PED had migrated proximally. The migration distance was $7.5 \mathrm{~mm}$. A second, overlapping $5 \times 30$ - $\mathrm{mm}$ PED was subsequently deployed beyond the first stent to bridge the neck of the aneurysm and disrupt the inflow jet. 

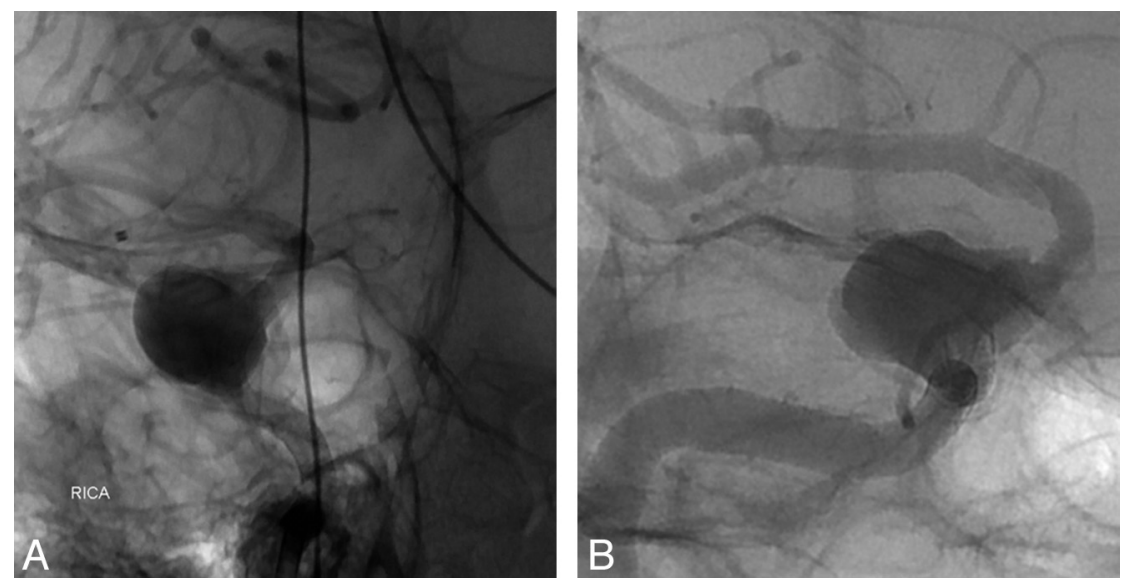

FIG 4. $A$, Angiogram showing the initial position of the PED; the distal end is at the clinoid segment. $B$, Angiogram showing proximal migration of the PED; the distal end of the device is now within the aneurysmal sac.

PED migration characteristics

\begin{tabular}{|c|c|c|c|c|}
\hline \multirow[b]{2}{*}{$\begin{array}{l}\text { Patient } \\
\text { No. }\end{array}$} & \multicolumn{2}{|c|}{$\begin{array}{l}\text { Length of PED Extending } \\
\text { to Aneurysm (mm) }\end{array}$} & \multirow{2}{*}{$\begin{array}{l}\text { Migration } \\
\text { Distance } \\
(\mathrm{mm})\end{array}$} & \multirow{2}{*}{$\begin{array}{l}\text { Migration of } \\
\text { Proximal or } \\
\text { Distal End } \\
\text { of PED? }\end{array}$} \\
\hline & $\begin{array}{l}\text { Proximal to } \\
\text { Aneurysm }\end{array}$ & $\begin{array}{l}\text { Distal to } \\
\text { Aneurysm }\end{array}$ & & \\
\hline 1 & 7 & 6 & 9 & Proximal \\
\hline 2 & 10 & 15 & 20 & Distal \\
\hline 3 & 15 & 3 & 5 & Distal \\
\hline 4 & 10 & 7 & $>10$ & Distal and proximal \\
\hline 5 & 13 & 3.5 & 7.5 & Distal \\
\hline
\end{tabular}

\section{DISCUSSION}

Several groups have recently reported their experience with the PED. In most series, remarkably high rates of aneurysm occlusion were achieved at follow-up with an acceptable risk for major complications. ${ }^{1-5}$ Some authors, based on favorable results from noncontrolled series, have even recommended the PED as a first-line option for unruptured aneurysms. ${ }^{3}$ Long-term safety results, however, are lacking and new complications have been emerging as neurointerventionalists accumulate experience with the device. The phenomenon of delayed migration or shortening of the PED has not been described in previously published series.

In this study, we have reported 5 cases of patients with spontaneous delayed migration or shortening of the PED (Table). In most cases, the PED migrated proximally (patients 2-5), and there was a significant difference in luminal diameter between inflow and outflow vessels. This may have produced a constant retrograde squeezing force at the distal end of the PED causing it to "watermelon-seed" backward into the aneurysm sac. Also, given the vertical position of the ICA, the weight of the device may have dragged the construct downward. The PED migration may also be at least in part the result of an "accordion effect," a phenomenon of foreshortening of a device that was possibly stretched during deployment. This is particularly true in patients 1,2 , and 5 where the PED moved from its initial position because of device shortening rather than migration. In patient 1 , the PED moved distally due to shortening because it was probably better anchored distally than it was proximally.

The incidence of spontaneous migration seems to be higher with the PED $(5 / 155,3.2 \%)$ than other self-expanding stents such as the Neuroform (Stryker Neurovascular, Fremont, California) (no cases previously reported) or Enterprise (Codman \& Shurtleff, Raynham, Massachusetts) (only 6 cases previously reported). ${ }^{8}$ The PED is particularly vulnerable to the phenomenon of spontaneous migration because it is a low-porosity stent, which facilitates the transmission of a force exerted at one end of the device to the other end. ${ }^{9-10}$ The migration of the PED is somewhat similar to the migration of Enterprise stents. Previous cases have involved Enterprise stents deployed from the midbasilar artery to the P1 segment of the posterior cerebral artery during treatment of basilar tip aneurysms. ${ }^{10-15}$ The migration was primarily attributed to the diameter difference between the basilar artery and the P1 segment of the posterior cerebral artery. Lubicz et $\mathrm{al}^{16}$ previously described the delayed migration of a Silk stent (Balt Extrusion, Montmorency, France) in a patient with a giant saccular carotid ophthalmic aneurysm. The patient had a massive SAH as a result of the migration and died.

Our present series shows that PED migration can occur both early and late after treatment. In patient 2, the migration occurred within 72 hours. Conversely, in patient 4 the migration likely occurred several months after the initial intervention, as suggested by the recurrence of the patient's symptoms at 5 months.

Although PED migration was uneventful in 3 patients, it was associated with fatal or severely disabling complications in 2 patients. In patient 2 , the distal end of the PED migrated proximally into the aneurysm sac, creating a direct jet of blood against the aneurysm wall and precipitating aneurysm rupture. This occurrence is further supported by the observation that the rupture site on the aneurysmal dome was directly facing the distal end of the device. In patient 1 , the patient presented with complete occlusion of the MCA, but it is not clear whether this event was related to the distal migration of the PED. Still, the device migration into the aneurysmal sac precluded any attempt at mechanical thrombolysis, and the procedure had to be aborted because of the high risk for iatrogenic aneurysm perforation (thus preventing potential neurologic recovery).

The following precautions should be taken to minimize the occurrence of spontaneous delayed PED migration or shortening, especially if there is a mismatch in diameter between inflow and outflow vessels:

1. Obtaining complete expansion of the PED with optimal vessel wall apposition. Accurate sizing of the PED is of utmost importance. DynaCT may be useful to confirm adequate apposition of the device to the vessel wall.

2. Using longer PEDs to prevent excessive foreshortening. In addition, the conveyance of retrograde forces is lower with longer stents. ${ }^{9,10}$ Increasing vessel coverage proximally and/or distally to the aneurysm may also be helpful. A second stent can be deployed to increase vessel coverage when needed. One could 
argue in retrospect that PED deployment was too proximal in patients 3 and 5 and was too distal in patient 1 .

3. Avoidance of dragging of the PED to prevent foreshortening. Instead of going in distally and dragging the device proximally into the landing zone, it would be preferable to go directly into the landing zone to detach and deploy the device.

4. Avoidance of stretching of the PED during deployment to prevent the "accordion effect."

5. Adjunctive aneurysm coiling, as this may prevent prolapse of the PED into the aneurysm for larger aneurysms.

We have recently applied these precautions, and we will assess in a future study whether they can lead to a significant decrease in the incidence of PED migration or shortening.

PED migration is best managed by placing additional PEDs to achieve complete coverage of the aneurysm and divert the blood flow away from the aneurysmal sac. A J-shaped guidewire is passed through the migrated stent and is navigated directly into the distal ICA. This is followed by deployment of 1 or more PEDs as necessary until the parent vessel is reconstructed. If the proximal part of the device has migrated into the aneurysmal sac and attempts to recatheterize the PED have failed, retrograde access of the PED is gained from the distal ICA through a microwire advanced from the basilar artery through the posterior communicating artery or from the contralateral ICA through the anterior communicating artery. ${ }^{17}$ Endovascular parent vessel deconstruction or surgical ligation, when tolerated, is an alternative strategy to manage PED migration. In the setting of subarachnoid hemorrhage, as in patient 2 , the goal of treatment is to obtain immediate occlusion of the aneurysmal sac and/or the parent vessel.

\section{CONCLUSIONS}

Spontaneous delayed migration or shortening of the PED is a serious and potentially fatal complication. The device may migrate proximally or distally and result in aneurysmal rupture or thrombotic events. The migration may occur both early and late after treatment. Obtaining complete expansion of the PED by using longer PEDs, increasing vessel coverage, using adjunctive aneurysm coiling, and avoiding dragging and stretching of the PED are important preventive measures. Neurointerventionalists should be aware of this undesirable phenomenon and take all necessary preventive measures. If any concern arises regarding the position of the PED, early imaging follow-up may be indicated.

Disclosures: Stavropoula Tjoumakaris—UNRELATED: Consultancy: Stryker. Pascal Jabbour-UNRELATED: Consultancy: ev3, CNV.

\section{REFERENCES}

1. Lylyk P, Miranda C, Ceratto R, et al. Curative endovascular reconstruction of cerebral aneurysms with the Pipeline embolization device: the Buenos Aires experience. Neurosurgery 2009;64:632-42; discussion 642-43; quiz N636

2. Saatci I, Yavuz K, Ozer C, et al. Treatment of intracranial aneurysms using the Pipeline flow-diverter embolization device: a single-center experience with long-term follow-up results. AJNR Am J Neuroradiol 2012;33:1436-46

3. Yu SC, Kwok CK, Cheng PW, et al. Intracranial aneurysms: midterm outcome of Pipeline embolization device-a prospective study in 143 patients with 178 aneurysms. Radiology 2012;265:893-901

4. McAuliffe $\mathrm{W}$, Wycoco $\mathrm{V}$, Rice $\mathrm{H}$, et al. Immediate and midterm results following treatment of unruptured intracranial aneurysms with the Pipeline embolization device. AJNR Am J Neuroradiol 2012;33:164-70

5. Nelson PK, Lylyk P, Szikora I, et al. The Pipeline embolization device for the intracranial treatment of aneurysms trial. AJNR Am J Neuroradiol 2011;32:34-40

6. Chitale R, Gonzalez LF, Randazzo C, et al. Single center experience with Pipeline stent: feasibility, technique, and complications. Neurosurgery 2012;71:679-91; discussion 691

7. O'Kelly CJ, Spears J, Chow M, et al. Canadian experience with the Pipeline Embolization device for repair of unruptured intracranial aneurysms. AJNR Am J Neuroradiol 2013;34:381-87

8. Prestigiacomo CJ. Stent-assisted coiling for aneurysms and the phenomenon of stent migration: is it worth it? J Neurointerv Surg 2010;2:351

9. Gao B, Malek AM. Possible mechanisms for delayed migration of the closed cell-designed Enterprise stent when used in the adjunctive treatment of a basilar artery aneurysm. AJNR Am J Neuroradiol 2010;31:E85-86

10. Lobotesis K, Gholkar A, Jayakrishnan V. Early migration of a self expanding intracranial stent: case report. Neurosurgery 2010;67: E516-17

11. Dashti SR, Fiorella D, Toledo MM, et al. Proximal migration and compaction of an Enterprise stent into a coiled basilar apex aneurysm: a posterior circulation phenomenon? J Neurointerv Surg 2010;2:356-58

12. Lavine SD, Meyers PM, Connolly ES, et al. Spontaneous delayed proximal migration of Enterprise stent after staged treatment of wide-necked basilar aneurysm: technical case report. Neurosurgery 2009;64:E1012; discussion E1012

13. Kelly ME, Turner RD, Moskowitz SI, et al. Delayed migration of a self-expanding intracranial microstent. AJNR Am J Neuroradiol 2008;29:1959-60

14. Rodriguez GJ, Maud A, Taylor RA. Another delayed migration of an Enterprise stent. AJNR Am J Neuroradiol 2009;30:E57

15. Khatri R, Rodriguez GJ, Siddiq F, et al. Early migration of a selfexpanding intracranial stent after the treatment of a basilar trunk aneurysm: report of a second case. Neurosurgery 2011;69:E513-15; author reply E515-17

16. Lubicz B, Collignon L, Raphaeli G, et al. Flow-diverter stent for the endovascular treatment of intracranial aneurysms: a prospective study in 29 patients with 34 aneurysms. Stroke 2010;41:2247-53

17. Hauck EF, Natarajan SK, Langer DJ, et al. Retrograde trans-posterior communicating artery snare-assisted rescue of lost access to a foreshortened Pipeline embolization device: complication management. Neurosurgery 2010;67:495-502 